\title{
NERVE SYMPTOMS IN VERTEBRAL RHEUMATISM
}

BY

\author{
C. W. BUCKLEY
}

Peripheral nerve symptoms are a common accompaniment of rheumatic diseases of the vertebral column and may be the first, or in the milder cases the only, manifestations. Frequently they are only of minor degree and may be dismissed by the doctor as " a touch of neuritis" and with a few tablets of aspirin; the stage at which treatment of the underlying cause is likely to be most effective is passed before its nature comes to be recognized. This may happen even in cases following an injury, as Walshe (1944) has pointed out, when the symptoms, as in those of rheumatic origin, may be slow and insidious in their development. Numbness often variable in character, sometimes clearing up altogether and then recurring without obvious cause, pins and needles, tingling sensations, and slight muscular weakness, shown perhaps by a tendency to drop things, may be noticed before any actual pain is experienced, which when it occurs may be worse at night and may also be influenced by heat or cold, characteristics which should suggest the possibility of a rheumatic cause. Disseminated sclerosis may be suspected and in the early stages it will be difficult to exclude, unless some rheumatic condition is discovered of a nature and degree adequate to produce the symptoms.

\section{Ankylosing Spondylitis}

Babinski reported three cases of lightning pains and loss of tendon reflexes in the lower limbs which gave rise to suspicion of tabes but proved to be due to ankylosing spondylitis; and Garrod (1913) attached importance to these nerve-root symptoms in ankylosing spondylitis, and mentions that some patients who apply for relief from pains of this origin are unconscious of the rigidity of their spines. He made the important observation that they are not due to narrowing of the foramina. Nathan (1916) studied the development of epidural and periradicular exudation in ankylosing spondylitis, and demonstrated that such exudations occurred in the early stages of the disease giving rise to sensory alterations of root distribution. As the inflammatory process subsided a varying amount of fibrosis remained, upon which the severity and permanence of the symptoms depended. These observations are of special interest in view of the fact that pains in the limbs, often fugitive in character but sometimes persistent, and without any obvious changes in the joints, are a feature of the early stages of the adolescent type of ankylosing spondylitis, often occurring for months or years before the appearance of symptoms directly referable to the spinal articulations. Nerve pains are much more frequent in the pre-ankylosing stage than when the spine becomes fixed, especially in the lower limbs, the usual site of onset in the adolescent type being in the lumbosacral region. Girdle pains in the lower intercostal region may, however, be due to spondylitis in those cases which while ankylosing in character are not adolescent in time of origin, and in which the maximum bony change in the early stages is in the lower thoracic and upper lumbar region.

\section{Vertebral Osteo-arthritis}

While peripheral nerve symptoms are to be met with in ankylosing spondylitis they are much more common in osteo-arthritis of the spine. Garcin and Deparis (1934) studied these nerve lesions especially and describe twelve cases. They point out that muscular atrophy of the Aran-Duchenne type, root atrophy of muscles especially in the leg and thigh, and symptoms indicating syringomyelia, lateral sclerosis, or chronic anterior polio-myelitis, may be set up by irritation and compression of nerve roots in their passage through the spinal foramina. They point out further that radiology in such cases often shows osteophytes in the region of the vertebræ from which the affected nerves emerge but that such lesions may be on the opposite side, and conclude that it is not the formed osteophytes that exert the pressure on the nerve roots but those in course of formation which have not yet undergone changes into bone opaque to $\mathrm{x}$-rays, or to inflammatory or congestive changes of rheumatic nature. At the date of their investigation, however, prolapses of the intervertebral disks had not received the recognition that has been attached to them of late, and it may be that some of the cases for which they offer the explanation referred to may have been cases of this nature.

The nerve symptoms may develop insidiously before obvious osteo-arthritic signs are present or may not appear till long after well-marked spinal osteophytes have been known to exist. They may be apparently induced by some intercurrent illness-influenza or even a common cold. Inflammatory disease in some neighbouring structure may set up local periostitis and thus pressure on the 
nerve root in its narrow canal or foramen. Burning or smarting pain is characteristic. The cervical spine seems especially prone to osteo-arthritis owing to the great mobility of this region; the intervertebral disks tend to lose their elasticity and shrink in middle life; this leads to greater play between the vertebral bodies and intermittent strain on the attachment of the muscles and ligaments, which in turn leads to the development of exostoses at their point of attachment. Frequently there is also atrophic change of a degenerative character in the vertebral bodies leading to some degree of collapse and secondary osteo-arthritic changes. As is usually the case in osteo-arthritis, fibrositis may also be present, and the crepitus often experienced in the neck, sometimes audibly, is generally due to fibrositis of the muscle sheaths and tendons and not to the vertebral articulations.

The sixth cervical is the largest of the nerve roots in this region and therefore the most liable to be affected by narrowing of the foramen; in consequence symptoms appear to be most frequently met with in the distribution of the musculo-spiral and median nerves. Careful search for sensory modifications in the area of the nerve roots concerned will help in diagnosis. Gunther and Kerr (1918) made a searching investigation into the nervous disturbances in hypertrophic osteo-arthritis of the spine in patients whose ages ranged from 34 to 72. Their observations upon pain in the region of abdominal organs and in the precordium arising from vertebral causes are of much interest and practical application. They stress the importance of pain, aching, and soreness brought on or aggravated by movement of the spinal column or by actions increasing the intraspinal pressure, such as sneezing or straining, and in all cases cutaneous sensory changes were commonly found. Among other important observations was the association of occipital and sometimes temporal headache with irritation of the first, second, or third cervical nerve roots, and they call attention to the frequency with which headache of this type is attributed to fibrositis of the occipital fascia, often in error. Nachlas (1934) has also described a syndrome of pseudoangina pectoris originating from demonstrable changes in the cervical spine.

Muscle symptoms are less frequently met with, since actual injury to the nerve fibres interfering with their conductivity, and not mere irritation, is necessary to cause any degree of atrophy. Fibrillation is, however, not uncommon, and atrophy, especially of the small hand-muscles, may occur; it is more liable to be unilateral than bilateral or may vary in degree between the two sides. Atrophy of this type may, however, be reflex in character, and is not uncommon in association, for instance, with osteo-arthritis of the carpal joint of the thumb. Reflexes may be affected and the deep reflexes of the arm should always be tested. Walshe (1944) has laid stress on the importance of unequal affection of the arm jerks as indicating the presence of a local cord lesion such as may result from bony changes in the vertebral canal, or from extrusion of an intervertebral disk.

Radiographic examination should always be carried out; the antero-posterior view is of little value as a rule except to show the presence of supernumerary ribs or abnormalities of the transverse processes, profile views are more useful and the three-quarter profile especially will often reveal obstruction of the foramina which might not otherwise be detected.

\section{Fibrositis}

Deep-seated fibrositis in the neighbourhood of the vertebral column may also give rise to peripheral nerve symptoms; this is not uncommon as a cause of sciatic pain, but may also affect the nerve roots in the cervical or dorsal region and give rise to brachialgia or intercostal neuritis. It may be the early stage of spondylitis, and Stockman (1926) states that "ossifying spondylitis" is due to the formation of new bone in chronically inflamed fibrous tissue. This, however, is not usually the case, though it may be noted that Oppenheimer (1938), who has made an extensive study of the various forms of spinal arthritis, stresses the fact that ossification of ligaments is a very common and totally uncharacteristic response to a great variety of intervertebral lesions, inflammatory, destructive, or traumatic. Simple fibrositis, however, with no inherent tendency to develop bony changes, may lead to the formation of exostoses, lipping of the edges of the vertebral bodies, and similar changes, vertebral osteo-arthritis being the end-result.

A case under the observation of the writer illustrates some of these features.

A woman of 50 gave a history of " lumbago" seven years earlier which had never entirely cleared up and had more recently been associated with pain in the left sciatic distribution; $x$-ray examination of the spine was negative. No infective focus of any kind could be discovered. The pain was chiefly on the left side, was made worse by walking and relieved by lying on that side. She could bend down nearly to touch the toes without pain; the spinal curvatures were normal; treading on any uneven surface caused pain to shoot up the back. She began to experience pain in the left intercostal and pectoral regions which was worse at night, and also pain on tilting the head over to the left side; she felt soreness in the arm with pins and needles sensation in the fingers. The biceps and supinator jerks were normal but the triceps jerk was elicited only with difficulty; the superficial abdominal reflexes were present; cutaneous sensation was normal. $\mathrm{X}$-ray examination showed a very slight degree of lipping of the anterior margins of the vertebral bodies in the lumbar and cervical regions but no thinning of the disks. Her general health was good, there were no indications of cardiac disease, weight was normal for her age. The symptoms improved to some extent with treatment but did not clear up entirely.

There seems little doubt that this was a case of fibrositis of the spinal muscles and ligaments. Pain in the left breast radiating down the arm must always give rise to suspicion of cardiac mischief, but in this case there was no other evidence of that and the history supported a diagnosis of fibrositis. 


\section{Diagnosis and Treatment}

Peripheral nerve pains, especially if variable in degree of severity or intermittent, should, unless there is an obvious cause, call for radiological examination of that area of the spine from which the nerves are derived. Particularly is this the case in adolescence or early adult life, when they may be due to ankylosing spondylitis. The early recognition of such a condition and its treatment by prolonged rest in bed are of the greatest importance, and it is unfortunate that in so many cases these early premonitory symptoms are disregarded. The sedimentation rate will help in diagnosis, but loss of density in the bones around the sacro-iliac and intervertebral articulations should always arouse suspicion; flattening of the lumbar spinal curve and the characteristic facies and gait may also be early signs. To deal in detail with the treatment of this disease is, however, outside our present scope. In later life special attention should be given in radiological examination to the profile and three-quarter profile views and to evidence of thinning of the intervertebral disks. It must be borne in mind that prolapse of the nucleus pulposus is not limited to the lumbar region but may be equally productive of symptoms in the cervical spine. Operative intervention is less often called for and rest in bed or the wearing of a suitable support for a time may result in relief of the pressure on the nerve root, as is sometimes the case in sciatica due to this condition.

Locally heat is often helpful and moist heat may be found more effective than the various rays which captivate the public imagination. Infra-red rays by their greater penetrative power have some advantages and may be combined with massage, which may be more effective if not too deep and directed rather to improving the local circulation than to breaking down local thickenings, which are not always responsible; very deep pressure may easily do harm. Ionization with histamine followed by light massage is another line of treatment frequently effective in relieving pain. Counterirritation by the electric cautery may be found useful, and deep x-rays provide a method which has given good results in spondylitis; it is of interest to note that some attribute their effect to counterirritation, which would justify the use of simpler methods of obtaining this result in the first instance. Manipulation may be successful, but should be attempted only by an orthopædic surgeon. Diathermy is a method which is frequently tried, but the fact that its effect is to raise the temperature of the deeper structures and thus to cause increased circulation and swelling of the tissues under its influence indicates that it may tend to increase the pain rather than give relief; as a preliminary to massage it may be worth a trial, but should not be persisted in unless definite relief is promptly experienced.

\section{REFERENCES}

Garcin, R. and Deparis, M. (1934). Rev. Méd. Franc., 15, 387 Garrod, Archibald (1913). System of Medicine by Allbutt and Rolleston, London, 3, 39

Gunther, L. and Kerr, W. J. (1929). Arch. Int. Med. 43, 212.

Nachlas, I. W. (1934). J. Amer. Med. Ass. 103, 323.

Nathan, P. W. (1916). Amer. J. Med. Sci. 152, 667

Oppenheimer, A. (1938). J. Bone Jt. Surg. 20, 285

Stockman, R. (1926). Edinb. Med. J., 33, 597.

Walshe, F. M. R. (1944). Lancet, 2, 173. 